\title{
Test of Generating Function and Estimation of Equivalent Radius in Some Weapon Systems and Its Stochastic Simulation
}

\author{
Famei Zheng \\ School of Mathematical Science, Huaiyin Normal University, Huai'an, China \\ E-mail:hysyzfm@163.com,16032@hytc.edu.cn,hssky10@163.com \\ Received November 16, 2011; revised December 6, 2011; accepted December 14, 2011
}

\begin{abstract}
We discuss three-dimensional uniform distribution and its property in a sphere; give a method of assessing the tactical and technical indices of cartridge ejection uniformity in some type of weapon systems. Meanwhile we obtain the test of generating function and the estimation of equivalent radius. The uniformity of distribution is tested and verified with $\omega^{2}$ test method on the basis of stochastic simulation example.
\end{abstract}

Keywords: Uniform Distribution in a Sphere, Weapon Systems, Generating Function, Equivalent Radius, Stochastic Simulation

\section{Introduction}

Uniform distribution is very important in the probability statistics, many scholars pay attention to it. The following questions have been explored: the estimate of interval length about uniform distribution in $[a, b][1,2]$, the estimate of regional area about two dimension uniform distribution in a rectangle [3], the estimate of cuboid volume about three dimension uniform distribution [4], the estimate of regional area about two-dimensional uniform distribution in a circle [5,6], estimate of radius on three-dimensional uniform distribution in a sphere [7]. In addition, many scholars get useful test statistics and limit theorems [8-12]. In this paper, basing on some articles [13-18], according to $t$ the indices of cartridge ejection uniformity in some type of weapon systems, we give the test of generating function and the estimation of equivalent radius by simulation example.

Definition 1 [7]. If $(X, Y, Z)$ is three-dimensional continuous random variable, its probability density function is

$$
f(x, y, z)= \begin{cases}\frac{3}{4 \pi R_{0}^{3}}, & (x, y, z) \in G, \\ 0 & (x, y, z) \notin G .\end{cases}
$$

where $G=\left\{(x, y, z) \mid x^{2}+y^{2}+z^{2}<R_{0}^{2}\right\}, R_{0}>0$, then we call that $(X, Y, Z)$ obeys uniform distribution in
$G=\left\{(x, y, z) \mid x^{2}+y^{2}+z^{2}<R_{0}^{2}\right\}$, recorded as $(X, Y, Z) \sim U(G)$.

Give a transformation

$\left\{\begin{array}{l}x=r \sin \varphi \cos \theta \\ y=r \sin \varphi \sin \theta\left(0<r<R_{0}, 0<\varphi<\pi, 0<\theta<2 \pi\right)(1.2) \\ z=r \cos \varphi\end{array}\right.$

The probability density function of three-dimensional r.v. $(R, \Phi, \Theta)$ is

$h(r, \varphi, \theta)=f(r \sin \varphi \cos \theta, r \sin \varphi \sin \theta, r \cos \varphi)\left|\frac{\partial(x, y, z)}{\partial(r, \varphi, \theta)}\right|$

in which $0<r<R_{0}, 0<\varphi<\pi, 0<\theta<2 \pi, \frac{\partial(x, y, z)}{\partial(r, \varphi, \theta)}$ is

Jacobi determinant of the transformation (1.2), and

$$
\begin{aligned}
& \frac{\partial(x, y, z)}{\partial(r, \varphi, \theta)}=\left|\begin{array}{lll}
\frac{\partial x}{\partial r} & \frac{\partial x}{\partial \varphi} & \frac{\partial x}{\partial \theta} \\
\frac{\partial y}{\partial r} & \frac{\partial y}{\partial \varphi} & \frac{\partial y}{\partial \theta} \\
\frac{\partial z}{\partial r} & \frac{\partial z}{\partial \varphi} & \frac{\partial z}{\partial \theta}
\end{array}\right| \\
& =\left|\begin{array}{ccc}
\sin \varphi \cos \theta & r \cos \varphi \cos \theta & -r \sin \varphi \sin \theta \\
\sin \varphi \sin \theta & r \cos \varphi \sin \theta & r \sin \varphi \cos \theta \\
\cos \varphi & -r \sin \varphi & 0
\end{array}\right| \\
& =r^{2} \sin \varphi
\end{aligned}
$$



is

Therefore the probability density function of $(R, \Phi, \Theta)$

$$
h(r, \varphi, \theta)=\left\{\begin{array}{lc}
\frac{3 r^{2} \sin \varphi}{4 \pi R_{0}^{3}}, 0<r<R_{0}, 0<\varphi<\pi, 0<\theta<2 \pi \\
0, & \text { otherwise }
\end{array}\right.
$$

Theorem 1. If the marginal density functions of $r . v$. $(R, \Phi, \Theta)$ about $R, \Phi, \Theta$ are $h_{1}(r), \quad h_{2}(\varphi), \quad h_{3}(\theta)$, then

$$
\begin{aligned}
& \text { 1) } h_{1}(r)= \begin{cases}\frac{3 r^{2}}{R_{0}^{3}}, & 0<r<R_{0}, \\
0, & \text { otherwise. }\end{cases} \\
& \text { 2) } h_{2}(\varphi)=\left\{\begin{array}{ll}
\frac{1}{2} \sin \varphi, 0<\varphi<\pi, \\
0, & \text { otherwise. }
\end{array} \text { 3) } h_{3}(\theta)= \begin{cases}\frac{1}{2 \pi}, & 0<\theta<2 \pi, \\
0, & \text { otherwise. }\end{cases} \right.
\end{aligned}
$$

Proof. According to (1.5) and the definition of marginal density function, we have

$$
\begin{aligned}
h_{1}(r) & =\int_{0}^{\pi} \int_{0}^{2 \pi} h(r, \varphi, \theta) \mathrm{d} \varphi \mathrm{d} \theta \\
& =\int_{0}^{\pi} \int_{0}^{2 \pi} \frac{3 r^{2} \sin \varphi}{4 \pi R_{0}^{3}} \mathrm{~d} \varphi \mathrm{d} \theta=2 \pi \times \frac{3 r^{2}}{4 \pi R_{0}{ }^{3}} \int_{0}^{\pi} \sin \varphi \mathrm{d} \varphi \\
& =2 \pi \times \frac{3 r^{2}}{4 \pi R_{0}{ }^{3}} \times 2=\frac{3 r^{2}}{R_{0}{ }^{3}},
\end{aligned}
$$

where $0<r<R_{0}$,

$$
\begin{aligned}
h_{2}(\varphi) & =\int_{0}^{R_{0}} \int_{0}^{2 \pi} h(r, \varphi, \theta) \mathrm{d} r \mathrm{~d} \theta=\int_{0}^{R_{0}} \int_{0}^{2 \pi} \frac{3 r^{2} \sin \varphi}{4 \pi R_{0}^{3}} \mathrm{~d} r \mathrm{~d} \theta \\
& =2 \pi \times \frac{3 \sin \varphi}{4 \pi R_{0}^{3}} \int_{0}^{R_{0}} r^{2} \mathrm{~d} r=\frac{1}{2} \sin \varphi,
\end{aligned}
$$

where $0<\varphi<\pi$,

$$
\begin{aligned}
h_{3}(\theta) & =\int_{0}^{R_{0}} \int_{0}^{\pi} h(r, \varphi, \theta) \mathrm{d} r \mathrm{~d} \varphi=\int_{0}^{R_{0}} \int_{0}^{\pi} \frac{3 r^{2} \sin \varphi}{4 \pi R_{0}^{3}} \mathrm{~d} r \mathrm{~d} \varphi \\
& =\frac{3}{4 \pi R_{0}^{3}} \int_{0}^{R_{0}} r^{2} \mathrm{~d} r \int_{0}^{\pi} \sin \varphi \mathrm{d} \varphi=\frac{3}{4 \pi R_{0}^{3}} \times \frac{1}{3} R_{0}^{3} \times 2 \\
& =\frac{1}{2 \pi},
\end{aligned}
$$

where $0<\theta<2 \pi$.

Corollary 1 [7]. If $r . v .(R, \Phi, \Theta)$ is defined by (1.5), then three r.v. $R, \Phi, \Theta$ are independent each other.

Corollary 2. If $r . v .(R, \Phi, \Theta)$ is defined by $(1.5)$, the marginal distribution function of $r . v .(R, \Phi, \Theta)$ about $R, \Phi, \Theta$ are $H_{1}(r), H_{2}(\varphi), H_{3}(\theta)$, then

$$
H_{1}(r)=\left\{\begin{array}{lr}
0, & r \leq 0 \\
\frac{r^{3}}{R_{0}^{3}}, & 0<r<R_{0} \\
1, & r \geq R_{0}
\end{array}\right.
$$$$
H_{2}(\varphi)=\left\{\begin{array}{lr}
0, & \varphi \leq 0 \\
\frac{1}{2}(1-\cos \varphi), & 0<\varphi<\pi, \\
1, & \varphi \geq \pi
\end{array}\right.
$$$$
H_{3}(\theta)=\left\{\begin{array}{lr}
0, & \theta \leq 0 \\
\frac{\theta}{2 \pi}, & 0<\theta<2 \pi \\
1, & \theta \geq 2 \pi
\end{array}\right.
$$

Proof. According to theorem 1, we can get it easily.

Corollary 3. If $E(R)=\mu, \operatorname{Var}(R)=\sigma^{2}$, then the probability of cartridges falling into a ball with radius $\mu$ is about $42.2 \%$, and the probability of cartridges falling into a sphere with radius $\mu+\sigma$ is about $84.0 \%$.

Proof. By the definition of Mathematical expectation, we have

$$
\begin{gathered}
\mu=E(R)=\int_{0}^{R_{0}} r h_{1}(r) \mathrm{d} r=\frac{3}{4} R_{0} \\
E\left(R^{2}\right)=\int_{0}^{R_{0}} r^{2} h_{1}(r) \mathrm{d} r=\int_{0}^{R_{0}} \frac{3 r^{4}}{R_{0}^{3}} \mathrm{~d} r=\frac{3}{5} R_{0}^{2}
\end{gathered}
$$

then

$$
\sigma^{2}=D(R)=E\left(R^{2}\right)-E^{2}(R)=\frac{3}{5} R_{0}^{2}-\frac{9}{16} R_{0}^{2}=\frac{3}{80} R_{0}^{2}
$$

and $\sigma=\frac{\sqrt{15}}{20} R_{0}$, then the probability of cartridges falling into a sphere with radius $\mu$ is about

$$
H_{1}(\mu)=H_{1}[E(R)]=H_{1}\left[\frac{3}{4} R_{0}\right] \approx 42.2 \%
$$

then the probability of cartridges falling into a sphere with radius $\mu+\sigma$ is about

$$
H_{1}(\mu+\sigma)=H_{1}\left(\frac{3}{4} R_{0}+\frac{\sqrt{15}}{20} R_{0}\right) \approx 84.0 \%
$$

\section{Test of Generating Distribution Function}

Usually there are $\chi^{2}$ test method, $\omega^{2}$ test method and Cole Moge Rove test method (K test method) [17] to test distribution function. Here, we use $\omega^{2}$ test method, we want to know the sub-sample is uniform distribution or not. Because the locations of any cartridges are ascertained by three-dimensional $r . v .(R, \Phi, \Theta)$, so we should 
test them one by one, test $R \sim H_{1}(r), \Phi \sim H_{2}(\varphi)$, $\Theta \sim H_{3}(\theta)$. We give testing hypotheses $H_{0}$

$$
H_{0}: F(x)=\Phi_{0}(x)
$$

where $F(x)$ is generating distribution function, $\Phi_{0}(x)$ is known distribution function, and $\varphi_{0}(x)$ is the derivative of $\Phi_{0}(x)$.

Tests for generating function should be independent, $y_{(1)}, y_{(2)}, \cdots, y_{(n)}$ is the sequent sub-sample of the test, under hypotheses $H_{0}$ is correct, the statistic

$$
n \omega^{2}=\frac{1}{12 n}+\sum_{i=1}^{n}\left[\Phi_{0}\left(y_{(i)}\right)-\frac{2 i-1}{2 n}\right]^{2}
$$

is Smirnov distribution. For the given confidence level $\alpha$, according to the Table 10 in [17], we obtain the boundary value $z_{\alpha}$ of $n \omega^{2}$, in which $P\left(n \omega^{2}>z_{\alpha}\right)=\alpha$. Then $\left(z_{\alpha}, \infty\right)$ is rejection region of the hypotheses $H_{0}$, when $n \omega^{2}>z_{\alpha}$, we reject $H_{0}$, if $n \omega^{2} \leq z_{\alpha}$, we should accept $H_{0}$.

\section{Estimation of Equivalent Radius}

On the supposition that $N$ is the number of cartridges from a shrapnel, $n$ is the actual observed number of cartridges within a certain region near the centre of dispersion. When calculating equivalent radius, we presume all the cartridges are found. The distances from any cartridges to the dispersion centre point $A$ are recorded as $r_{i}(i=1,2, \cdots, n)$, let $\bar{r}=\frac{1}{n} \sum_{i=1}^{n} r_{i}$. According to the properties of density function $h_{1}(r)$, we know that $R$ obeys uniform distribution in a ball with radius

$$
\begin{gathered}
r_{n 0}\left(0<r_{n 0}<R_{0}\right), E(R)=\frac{3}{4} r_{n 0} \text { (by 1.6), owing to } \\
E(\bar{r})=E\left(\frac{1}{n} \sum_{i=1}^{n} r_{i}\right)=\frac{1}{n} \sum_{i=1}^{n} \int_{0}^{r_{n} 0} r_{i} \frac{3 r_{i}^{2}}{r_{n 0}^{3}} d r_{i}=\frac{3}{4} r_{n 0}
\end{gathered}
$$

so $\hat{r}_{n 0}$ is a unbiased estimate of $r_{n 0}$ [7]. on the basis of the properties of distribution function, let $\varphi=\pi, \theta=2 \pi$, we have $H(r)=H(r, \varphi, \theta) \mid \begin{aligned} & \varphi=\pi \\ & \theta=2 \pi\end{aligned}$, let $N \rightarrow \infty,(N$ is amount of test cartridges ), then

$$
\lim _{N \rightarrow \infty} H(r, \varphi, \theta) \mid \begin{aligned}
& \varphi=\pi \\
& \theta=2 \pi
\end{aligned}=\lim _{N \rightarrow \infty} \frac{n}{N}=\frac{r_{n 0}^{3}}{R_{0}^{3}}
$$

Therefore let $\hat{R}_{0}=\sqrt{\frac{N}{n}} \hat{r}_{n 0}$, that is $\hat{R}_{0}=\frac{4}{3 n} \sqrt{\frac{N}{n}} \sum_{i=1}^{n} r_{i}$, and

$$
D\left(\hat{R}_{0}\right)=\frac{16 N}{9 n^{2}} D\left(r_{i}\right)
$$

As well as by (1.7), $D\left(r_{i}\right)=\frac{3}{80} r_{n 0}^{2}$, substitute into
(3.3), we obtain

$$
\begin{aligned}
& D\left(\hat{R}_{0}\right)=\frac{16 N}{9 n^{2}} D\left(r_{i}\right)=\frac{16 N}{9 n^{2}} \times \frac{3}{80} r_{n 0}^{2}=\frac{N r_{n 0}^{2}}{15 n^{2}}, \\
& \sigma\left(\hat{R}_{0}\right)=\frac{\sqrt{15 N} r_{n 0}}{15 n}
\end{aligned}
$$

Based on Formula (3.4), if $N$ is large enough, when $n \rightarrow N, \sigma\left(\hat{R}_{0}\right)$ becomes small enough. In order to decrease estimating error of equivalent radius $R_{0}$, sample size is large enough.

\section{Stochastic Simulation}

In order to verify the correctness of above methods, we give a simulation example. For some type of weapon systems, assuming the tactical technical requirements, the cartridges from single shrapnel should be more evenly scattered in a ball with equivalent radius $(120 \pm$ 20) $\mathrm{m}$, launching a shrapnel, the number of cartridges is $N=400$, measuring the coordinates of one hundred cartridges near the dispersion centre $(n=100)$, they were produced by computer simulation basing on uniform requirements in a sphere, i.e. $(r, \varphi, \theta)$ were produced by stochastic simulation according to the following formulas

$$
\begin{gathered}
r=\sqrt[3]{H_{1}(r)} r_{n 0}, \varphi=\arccos \left[1-2 H_{2}(\varphi)\right], \\
\theta=2 \pi H_{3}(\theta)
\end{gathered}
$$

where, $r_{n 0}=60, H_{1}(r), H_{2}(\varphi), H_{3}(\theta)$ are random number produced by stochastic simulation in $(0,1)$, coordinates for cartridges as below Table 1, and the MATLAB program as below,

$>$ clear

for $\mathrm{k}=1: 100$

$$
\begin{aligned}
\mathrm{r}=\operatorname{rand}(1,3) ; & \\
\mathrm{x} & =60 * \operatorname{sqrt}(\mathrm{r}(1)) ; \\
\mathrm{y} & =\operatorname{acos}(-2 * \mathrm{r}(2)+1) ; \\
\mathrm{z} & =2 * \mathrm{pi} * \mathrm{r}(3) ;
\end{aligned}
$$

$[\mathrm{x}, \mathrm{y}, \mathrm{z}]$

end

$>>$ clear

for $\mathrm{k}=1: 100$

$\mathrm{a}=\left(\mathrm{x}^{\wedge} 2-((2 * \mathrm{k}-1) / 200)\right) \wedge 2$;

$\mathrm{b}=\left(\mathrm{y}^{\wedge} 2 / 3600-((2 * \mathrm{k}-1) / 200)\right)^{\wedge} 2$;

$\mathrm{c}=\left(\mathrm{z}^{\wedge} 2 / 3600-((2 * \mathrm{k}-1) / 200)\right)^{\wedge} 2$;

$[\mathrm{a}, \mathrm{b}, \mathrm{c}]$

end

$>$ clear

for $\mathrm{k}=1: 100$

$\mathrm{s}=1 / 1200+\operatorname{sum}(\mathrm{a})$;

$\mathrm{u}=1 / 1200+\operatorname{sum}(\mathrm{b})$;

$\mathrm{v}=1 / 1200+\operatorname{sum}(\mathrm{c})$;

[s u v]

End 
Table 1. Polar coordinates points of fall for cartridges.

\begin{tabular}{|c|c|c|c|c|c|c|c|c|c|c|c|}
\hline \multicolumn{3}{|c|}{$(\mathrm{r} / \mathrm{m}, \varphi / \mathrm{rad}, \theta / \mathrm{rad})$} & \multicolumn{3}{|c|}{$(\mathrm{r} / \mathrm{m}, \varphi / \mathrm{rad}, \theta / \mathrm{rad})$} & \multicolumn{3}{|c|}{$(\mathrm{r} / \mathrm{m}, \varphi / \mathrm{rad}, \theta / \mathrm{rad})$} & \multicolumn{3}{|c|}{$(\mathrm{r} / \mathrm{m}, \varphi / \mathrm{rad}, \theta / \mathrm{rad})$} \\
\hline 57.8262 & 1.1283 & 0.0936 & 56.9007 & 1.7096 & 0.0811 & 55.0439 & 0.4464 & 5.2025 & 47.7960 & 0.6985 & 5.5996 \\
\hline 58.8251 & 1.3574 & 1.8108 & 33.8961 & 1.4923 & 1.9503 & 43.4513 & 2.1067 & 5.7655 & 33.1923 & 2.1410 & 3.0668 \\
\hline 41.6750 & 1.5768 & 5.1315 & 19.1279 & 1.4612 & 4.8952 & 45.6907 & 2.4810 & 0.7106 & 48.3936 & 1.3183 & 6.2367 \\
\hline 45.5454 & 2.0309 & 6.1921 & 54.1207 & 0.6013 & 1.9308 & 47.0793 & 1.1287 & 5.1026 & 51.7387 & 2.2742 & 2.3455 \\
\hline 46.6895 & 1.1728 & 0.1093 & 48.7537 & 1.4576 & 5.8226 & 50.8327 & 1.0500 & 5.7070 & 15.1818 & 0.4352 & 3.3389 \\
\hline 31.8301 & 0.6831 & 5.1484 & 39.0650 & 1.3001 & 4.2644 & 33.6245 & 2.6167 & 0.9827 & 56.5426 & 1.7679 & 1.1391 \\
\hline 30.8478 & 1.4572 & 3.9025 & 43.0160 & 1.1647 & 0.4668 & 7.5595 & 0.7407 & 0.7672 & 55.7802 & 2.6869 & 3.1535 \\
\hline 48.6323 & 1.5043 & 3.5198 & 14.0717 & 2.3512 & 0.4442 & 55.4707 & 2.6500 & 4.7922 & 53.2184 & 1.1347 & 2.6528 \\
\hline 53.9209 & 0.2431 & 1.5331 & 57.6967 & 2.1165 & 0.0748 & 48.0499 & 1.9865 & 4.5352 & 46.3803 & 2.4616 & 4.1494 \\
\hline 44.1604 & 1.9052 & 5.1648 & 57.1905 & 2.6896 & 1.4275 & 35.8438 & 2.3398 & 4.0941 & 26.1303 & 0.6488 & 4.2330 \\
\hline 42.6194 & 2.0355 & 1.6537 & 38.0081 & 1.6869 & 3.2440 & 28.1616 & 0.9503 & 4.7375 & 56.1754 & 0.5168 & 6.0149 \\
\hline 39.4989 & 1.1188 & 4.7350 & 49.7335 & 0.2389 & 2.8790 & 32.3887 & 1.4809 & 4.1670 & 34.6740 & 1.0105 & 1.2057 \\
\hline 57.2477 & 1.0742 & 4.1444 & 32.5254 & 1.7644 & 4.4183 & 44.4830 & 0.5776 & 5.5512 & 45.8214 & 2.6183 & 0.6987 \\
\hline 51.3376 & 2.0009 & 1.3452 & 50.4480 & 2.2554 & 3.6600 & 44.4939 & 2.3493 & 1.7103 & 14.1080 & 0.5078 & 3.5506 \\
\hline 37.3488 & 2.1746 & 3.7831 & 41.5086 & 2.8378 & 3.1994 & 22.4951 & 1.6951 & 2.6352 & 40.5506 & 1.0797 & 6.0897 \\
\hline 59.5588 & 2.9061 & 3.8007 & 52.2026 & 0.9810 & 0.4668 & 58.8127 & 1.2010 & 1.3383 & 57.3967 & 3.0969 & 0.1489 \\
\hline 51.7199 & 1.5174 & 4.1438 & 57.1376 & 1.9904 & 1.2139 & 31.8798 & 1.3179 & 0.2237 & 56.5065 & 0.9570 & 5.4676 \\
\hline 36.7509 & 2.5075 & 1.1523 & 49.6781 & 1.6150 & 2.3851 & 43.6260 & 2.3973 & 0.5102 & 41.5920 & 1.5676 & 0.1690 \\
\hline 52.7956 & 1.4728 & 3.9992 & 59.6074 & 2.6175 & 1.7367 & 40.6554 & 1.3123 & 5.3445 & 57.5123 & 1.1385 & 3.2641 \\
\hline 52.3921 & 2.2256 & 1.0700 & 55.5081 & 2.0117 & 4.8437 & 33.1399 & 0.5499 & 2.1375 & 46.9686 & 1.9237 & 1.2083 \\
\hline 30.7568 & 2.2887 & 3.3904 & 32.0629 & 0.9956 & 1.9723 & 57.8563 & 0.9268 & 2.9292 & 49.4790 & 2.7288 & 4.4969 \\
\hline 16.9386 & 0.8401 & 3.9169 & 56.4546 & 1.4698 & 4.0099 & 41.1545 & 0.4487 & 5.7416 & 51.0491 & 2.1332 & 1.5752 \\
\hline 38.4027 & 1.3570 & 4.3096 & 34.6080 & 0.8558 & 6.1990 & 54.1232 & 1.7046 & 1.4363 & 52.2896 & 1.9094 & 5.8679 \\
\hline 29.3040 & 1.6124 & 4.2556 & 51.6795 & 2.7865 & 3.1598 & 44.6063 & 0.7133 & 5.4161 & 51.0684 & 0.7404 & 0.8621 \\
\hline 24.6450 & 2.0222 & 5.5091 & 52.4759 & 1.2780 & 5.9546 & 44.2010 & 1.6150 & 4.1255 & 52.8935 & 0.6280 & 3.2773 \\
\hline
\end{tabular}

According to the data in Table 1 and methods in this paper, using above MATLAB program, we obtain

$$
\begin{aligned}
& n \omega_{r}^{2}=0.0888, n \omega_{\varphi}^{2}=0.0425, n \omega_{\theta}^{2}=0.0749 \\
& \hat{R}_{0}=117.4909, \sigma\left(\hat{R}_{0}\right)=3.0984
\end{aligned}
$$

Take conspicuous level $\alpha=10 \%$, seeing the Table 10 in [17], we obtain the boundary value $z_{\alpha}=0.3472$ for $n \omega^{2}$. Because $n \omega_{r}^{2}, n \omega_{\varphi}^{2}, n \omega_{\theta}^{2}$ are less than 0.3472, so we consider that cartridges obeys uniform distribution in a sphere.

\section{References}

[1] G. S. Chen, "Interval Estimate of the Interval Length on Uniform Distribution," Pure and Applied Mathematics, Vol. 22, No. 3, 2006, pp. 349-354.

[2] H. B. Zhang, "The Shortest Confidence Interval of the Interval Length on Uniform Distribution," Journal of Xiaogan University, Vol. 27, No. 3, 2007, pp. 52-55.

[3] Z. J. Liu, "Estimate of Rectangle Region Area on Two
Dimensional Uniform Distribution," College Mathematics, Vol. 23, No. 4, 2007, pp. 155-159.

[4] Z. J. Liu, "Estimate of Cuboid Volume on Three-Dimensional Uniform Distribution," Statistics and Decision, No. 5, 2007, pp. 23-24.

[5] Z. X. Wang, "Parameter Estimation of Two-Dimensional Uniform Distribution in a Circle," College Mathematics, Vol. 24, No. 2, 2008, pp. 150-152.

[6] W. Q. Jin, D. S. Cui and B. Deng, "On the Testing and Estimation of Uniform Distribution in a Circle," Acta Armamentarii, Vol. 22, No. 4, 2001, pp. 468-472.

[7] F. M. Zheng, "Estimate of Radius on Three-Dimensional Uniform Distribution in a Sphere," Mathematics in Practice and Theory, Vol. 40, No. 14, 2010, pp. 166-170.

[8] Y. X. Liu and P. Cheng, "Uniform Distribution of Ball PPCM Test Statistic on Dimension and Sample Size Berry-Esseen Boundary and LIL," China Science Bulletin, Vol. 43, No. 13, 2005, pp. 1452-1453.

[9] S. R. Xie, "Two Types of Stay Limit Theorems of NonStationary Gaussian Process," Science in China, Series A, Vol. 23, No. 4, 1993, pp. 369-376. 
[10] Z. S. Hu and C. Su, "Limit Theorems for the Number and Sum of Near-Maxima for Medium Tails," Statistics \& Probability Letters, Vol. 63, No. 3, 2003, pp. 229-237. doi:10.1016/S0167-7152(03)00085-3

[11] Y. Qi, "Limit Distributions for Products of Sums," Statistics \& Probability Letters, Vol. 62, No. 1, 2007, pp. 93100. doi:10.1016/S0167-7152(02)00438-8

[12] G. Rempala and J. Wesolowski, "Asymptotics for Products of Sums and U-Statistics," Electronic Communications in Probability, Vol. 7, No. 7, 2002, pp. 47-54.

[13] K. T. Fang, J. Q. Fan, H. Jin, et al., "Statistical Analysis of Directional Date," Journal of Application of Statistics and Management, Vol. 9, No. 2, 1990, pp. 59-65.

[14] M. D. Troutt, W. K. Pang and S. H. Hou, "Vertical Density Representation and Its Applications," World Scien- tific Publishing Co. Pte. Ltd, Singapore, 2004. doi:10.1142/9789812562616

[15] Z. S. Wei, "Probability Theory and Mathematical Statistics," Higher Education Press, Beijing, 1983.

[16] S. M. Berman, "Extreme Sojourns of a Gaussian Process with a Point of Maximum Variance," Probability Theory and Related Fields, Vol. 74, 1987, pp. 113-124. doi:10.1007/BF01845642

[17] C. P. Pan and Z. J. Han, "Probability and Statistic of Weapon Test," National Defence Industry Press, Beijing, 1979.

[18] N. I. Sidnyaev and K. S. Andreytseva, "Independence of the Residual Quadratic Sums in the Dispersion Equation with Noncentral $\chi^{2}$-Distribution," Applied Mathematics, Vol. 2, No. 2, 2011, pp. 1303-1308. 